\section{RMD Open}

Rheumatic \&

Musculoskeletal Diseases

\title{
No evidence for a direct role of HLA-B27 in pathological bone formation in axial SpA
}

\author{
Barbara Neerinckx, ${ }^{1,2}$ Simon Kollnberger, ${ }^{3}$ Jacqueline Shaw, ${ }^{4}$ Rik Lories ${ }^{1,2}$
}

To cite: Neerinckx B, Kollnberger S, Shaw J, et al. No evidence for a direct role of HLA-B27 in pathological bone formation in axial SpA. RMD Open 2017;3:e000451. doi:10.1136/ rmdopen-2017-000451

- Prepublication history for this paper is available online. To view these files, please visit the journal online (http://dx.doi. org/10.1136/rmdopen-2017000451).

Received 9 February 2017 Revised 19 April 2017 Accepted 2 May 2017

Published Online

First 27 May 2017

\section{CrossMark}

${ }^{1}$ Laboratory of Tissue Homeostasis and Disease, Skeletal Biology and Engineering Research Center, Leuven, Belgium

${ }^{2}$ Division of Rheumatology, UZ Leuven, Leuven, Belgium ${ }^{3}$ Cardiff Institute of Infection and Immunity, Cardiff University, Cardiff, UK

${ }^{4}$ Nuffield Department of Orthopaedics, Rheumatology and Musculoskeletal Sciences, Botnar Research Centre, 0xford University, Oxford, UK

Correspondence to Professor Rik Lories; rik.lories@ uz.kuleuven.be

\section{ABSTRACT}

Objective The strong genetic association between HLA-B27 and ankylosing spondylitis has been known for over 40 years. HLA-B27 positivity is possibly associated with severity of ankylosis. We studied the in vitro and in vivo impact of HLA-B27 in models of chondrogenesis and osteogenesis.

Methods Different in vitro differentiation systems were used to mimic endochondral and direct bone formation. ATDC5 cells and primary human periosteum-derived cells (hPDCs) were transduced with lentiviral vectors expressing HLA-B27 or HLA-B7. These cells and limb bud cells (from HLA-B27 transgenic and wild-type (WT) mice) were cultured in micromasses. To study direct osteogenesis in hPDCs, cells were cultured as monolayers and stimulated with osteogenic media. Chondrogenesis (COL2, ACAN, COL10) and osteogenesis (OSC, ALP, RUNX2) marker expression was studied by quantitative RT-PCR. Colorimetric tests were performed to measure proteoglycans, mineralization and collagens. Collagen antibody-induced arthritis (CAIA) was induced in HLA-B27 transgenic and WT mice. Clinical scoring and $\mu C T s$ were performed. Statistical analyses were performed by twoway ANOVA.

Results There was no difference in chondrogenesis markers or in colorimetric tests between $\mathrm{HLA}-\mathrm{B} 27^{+}$ and $\mathrm{HLA}-\mathrm{B} 7^{+}$micromasses. Expression of osteogenesis markers and Alizarin red staining was comparable in the $\mathrm{HLA}-\mathrm{B} 27^{+}$and the HLA-B7 $7^{+} \mathrm{hPDCs}$ in monolayers. HLA-B27 transgenic mice showed more severe arthritis compared with WT mice in the CAIA model. $\mu$ CT analysis showed no increased bone formation in HLA-B27 transgenic mice.

Conclusion HLA-B27 seems to enhance joint inflammation in the CAIA model. We could not document a direct effect of HLA-B27 on chondrogenesis or osteogenesis.

\section{INTRODUCTION}

Ankylosing spondylitis (AS) is the most well-known form of axial spondyloarthritis (axSpA), a group of common inflammatory chronic musculoskeletal diseases. Both inflammation and structural damage to the skeleton determine the long-term outcome in patients with AS. ${ }^{1}$ The former presents as

\section{Key messages}

What is already known about this subject?

- HLA-B27 is possibly associated with the severity of progressive ankylosis in patients with axial SpA.

- The exact role of HLA-B27 in the pathogenesis of axial $\mathrm{SpA}$ is unknown.

What does this study add?

- This study demonstrates that HLA-B27 does not play a direct role in chondrogenesis and osteogenesis but possibly rather an indirect role by influencing inflammation.

How might this impact on clinical practice?

- A tight control of inflammation in HLA-B27-positive axial SpA patients might prevent progression to ankylosis.

osteitis, enthesitis or synovitis and results in pain, swelling and associated loss of function. The latter is characterised by progressive ankylosis of the sacroiliac joints and spine and with new cartilage and bone formation leading to loss of spinal mobility. ${ }^{2} \mathrm{AS}$ is a paradigm for complex disorders with both genetic and environmental factors playing a role in disease onset and progression. ${ }^{3}$ The relationship between AS and the HLA-B27 gene is striking ${ }^{45}$ and remains the strongest association between an immune-mediated disease and the major histocompatibility complex (MHC) known to date. ${ }^{6}$ However, despite 40 years of research, it remains unclear how HLA-B27 really contributes to AS. ${ }^{7}$

Radiographic progression in patients with AS is highly variable between individual patients. Within the patient group with AS, the presence of HLA-B27 is hypothesised as a risk factor for more severe radiographic disease progression although data from large studies are somewhat conflicting. A long-term study, with a follow-up of 12 years, showed more severe radiographic progression in 
HLA-B27-positive men. ${ }^{8}$ In addition, accelerated early structural disease progression was shown in HLA-B27-positive AS patients as compared with HLA-B27-negative AS patients. ${ }^{910}$ However, in another large study, no association between presence of HLA-B27 and radiographic progression over 4 years could be demonstrated. ${ }^{11}$ Within the group of patients with axSpA, the proportion of HLA-B27 $7^{+}$patients is higher in the AS group as compared with the non-radiographic axSpA group. ${ }^{12}$ The eventual impact of HLA-B27 on structural disease progression could be explained by more severe inflammation triggering ankylosis in the HLA-B27-positive patients or by a direct molecular effect of HLA-B27 on the bone formation process.

In this study, we investigated the potential direct role of HLA-B27 in new cartilage and bone formation in AS by using different in vitro and in vivo models.

\section{MATERIALS AND METHODS}

\section{Cell culture experiments}

Lentiviral vectors

Lentiviral vectors expressing GFP-linked HLA-B27 (pCH-SFFV-B27-ires-GFP) and HLA-B7 (pCH-SFFV-B7ires-GFP) were produced by the Leuven Viral Vector Core. HLA-B27 and HLA-B7 plasmids were pHR-SIN-BN (HLAB27 and HLA-B7 cDNAS were cloned between BamHI and NotI sites). After production, functional titres were determined for both vectors and were $1.82 \times 10^{8} \mathrm{TU} / \mathrm{mL}$ for the HLA-B27 viral vector and $1.75 \times 10^{8} \mathrm{TU} / \mathrm{mL}$ for the HLA-B7 viral vector.

\section{ATDC5 cells}

ATDC5 cells were transduced using the HLA-B27-expressing and HLA-B7-expressing lentiviral vectors. ATDC5 cells were cultured at a concentration of $2 \times 10^{5}$ cells $/ \mathrm{mL}$ in a 12 -well plate until $90 \%$ confluence. Forty microlitres of viral vector was subsequently added to the cells for 48 hours. After incubation, cells were trypsinized and expanded. Transduced ATDC5 cells were cultured as 3D micromasses for 21 days to stimulate chondrogenesis. Micromasses were established and processed as earlier described.$^{13}$ In brief, trypsinized cells were resuspended in DMEM medium (Gibco Life Technologies, Ghent, Belgium) at a concentration of $2 \times 10^{7}$ cells $/ \mathrm{mL}$. One drop of $10 \mu \mathrm{L}$ of this cell suspension was placed in a well of a standard 24-well culture plate. Cells were allowed to adhere for 2 hours at $37^{\circ} \mathrm{C}$; subsequently, $0.5 \mathrm{~mL}$ medium was added to each well. For induction of chondrogenesis, micromasses were cultured in DMEM/F12 medium (Gibco Life Technologies) containing 1\% antibiotic-antimycotic (AB) (Gibco) and 5\% FBS (Gibco), supplemented with an ITS premix (containing $10 \mu \mathrm{g} /$ $\mathrm{mL}$ insulin, $5 \mu \mathrm{g} / \mathrm{mL}$ human transferrin and $3 \times 10^{-8} \mathrm{M}$ sodium selenite) (Gibco) and $5 \mu \mathrm{g} / \mathrm{mL}$ human transferrin (Sigma-Aldrich, Diegem, Belgium) for 2 weeks. From day 14 until day 21, a mineralization phase was induced using $\alpha$-MEM medium (Gibco) containing $1 \%$
$\mathrm{AB}$ and 5\% FBS and supplemented with ITS premix, $5 \mu \mathrm{g} / \mathrm{mL}$ human transferrin, $7 \mathrm{mM} \beta$-glycerophosphate (Sigma-Aldrich) and $50 \mu \mathrm{g} / \mathrm{mL}$ ascorbic acid-2-phosphate (Sigma-Aldrich). Medium was replaced daily. Each condition was performed in triplicate.

\section{Human periosteal derived cells}

Human periosteal derived cells (hPDCs) were isolated and cultured as described previously ${ }^{14}$ with the approval of the ethical committee of UZ Leuven (ML 7861). In short, periosteal biopsies $\left(0.5 \mathrm{~cm}^{2}\right)$ were harvested from the medial side of the proximal tibia of male and female adolescent and adult patients during total knee replacement surgery or distraction osteogenesis. The periosteum was stripped from the tibia with a periosteal lifter. Specimens were transported in growth medium (GM) consisting of high-glucose DMEM supplemented with $1 \%$ $\mathrm{AB}$ and $10 \%$ FBS. The biopsies were digested overnight at $37^{\circ} \mathrm{C}$ in $0.2 \%$ type IV collagenase (Invitrogen, Merelbeke, Belgium) in GM. Subsequently, periosteal cells were collected by centrifugation and seeded in T25 flask in GM. Non-adherent cells were removed after 5 days by changing the medium, and the remaining cells were expanded in monolayer in GM. On confluence, hPDCs were trypsin-released $(0.25 \%$ trypsin, $1 \mathrm{mM}$ EDTA; Invitrogen) and re-plated with a seeding density of 5000 cells $/ \mathrm{cm}^{2}$. From passage 2, hPDCs from different donors were pooled to reduce noise from genetic variability. For cryopreservation, hPDCs were suspended in DMEM with $20 \%$ FBS and 10\% DMSO (Sigma, Bornem, Belgium) and stored in liquid nitrogen. Cells were thawed, sub-cultured and subsequently transduced with the HLA-B27 and HLA-B7 lentiviral vectors. hPDCs were cultured at a concentration of $1 \times 10^{5}$ cells $/ \mathrm{mL}$ in a 24-well plate until $90 \%$ confluence. Twenty-five microlitres of viral vector was subsequently added to the cells for 48 hours. After incubation, cells were trypsinized and expanded. Transduced hPDCs were cultured as 3D micromasses to study chondrogenesis. Trypsinized cells were resuspended in DMEM/F12 medium at a concentration of $1.5 \times 10^{7}$ cells $/ \mathrm{mL}$. One drop of $10 \mu \mathrm{L}$ of this cell suspension was placed in a well of a standard 24-well culture plate. Cells were allowed to adhere for 2 hours at $37^{\circ} \mathrm{C}$; subsequently, $0.5 \mathrm{~mL}$ medium was added to each well. Standard medium used was DMEM/F12 supplemented with $1 \% \mathrm{AB}, 2 \% \mathrm{FBS}$ and $20 \mu \mathrm{M}$ Y-27632 Rock1 inhibitor (Merck Millipore, Overijse, Belgium). After 48 hours, standard medium was replaced by chondrogenic medium containing DMEM/F12 medium supplemented with 1\% $\mathrm{AB}, 2 \%$ FBS, ITS+ premix (Corning, New York, USA), $100 \mathrm{nM}$ dexamethasone (Sigma-Aldrich), $20 \mu \mathrm{M}$ Y-27632 Rock1 inhibitor, $50 \mu \mathrm{g} / \mathrm{mL}$ ascorbic acid-2-phosphate, $40 \mu \mathrm{g} / \mathrm{mL}$ proline (PeproTech, London, UK) and $10 \mathrm{ng} /$ mL TGF $\beta 1$ (PeproTech). Micromasses were cultured for 14 days and medium was replaced twice a week. Each condition was performed in triplicate.

To study osteogenesis in hPDCs, transduced cells were cultured in monolayers for 28 days. Twenty-fourhours 
after seeding, osteogenic medium (DMEM (Gibco) supplemented with $1 \% \mathrm{AB}, 10 \% \mathrm{FBS}, 100 \mathrm{nM}$ dexamethasone, $50 \mu \mathrm{g} / \mathrm{mL}$ ascorbic acid-2-phosphate and $10 \mathrm{mM}$ $\beta$-glycerophosphate) was added to the cells. Medium was replaced twice a week. Each condition was performed in triplicate.

\section{Limb bud cells}

HLA-B27 transgenic male C57BL/6 mice (a gift from Prof. J. Taurog, Texas, USA) ${ }^{15}$ were crossed with wild-type (WT) C57BL/6 female mice. At day E11.5, the (pregnant) mouse was sacrificed. Limb bud cells were derived from the HLA-B27 transgenic and WT $\mathrm{C} 57 \mathrm{Bl} / 6$ mice embryos. After washing the cells with phosphate-buffered saline (PBS) (Lonza, Verviers, Belgium) and disintegrating them with TripLE (Gibco), maintenance medium (40\% DMEM, 60\% DMEM/F12 supplemented with 1\% $\mathrm{AB}$ and $10 \% \mathrm{FBS}$ ) was added to a final concentration of $2 \times 10^{7}$ cells $/ \mathrm{mL}$. One drop of $10 \mu \mathrm{L}$ of this cell suspension was placed in a well of a standard 24-well culture plate. The cells were allowed to adhere for 2 hours at $37^{\circ} \mathrm{C}$; subsequently, $0.5 \mathrm{~mL}$ of chondrogenic medium (40\% DMEM, 60\% DMEM/F12 supplemented with 1\% $\mathrm{AB}, 10 \% \mathrm{FBS}, 1 \mathrm{mM} \beta$-glycerophosphate and $0.25 \mathrm{mM}$ ascorbic acid-2-phosphate) was added to each well. Medium was changed twice a week.

\section{Flow cytometry}

Fluorescence-activating cell sorting (FACS) analysis was used to determine surface expression of HLA-B27 on ATDC5 cells and hPDCs after transduction. Cells were incubated with an anti-HLA-B27 antibody (MAB1285; Millipore, Overijse, Belgium) for $30 \mathrm{~min}$. This antibody is not specific for HLA-B27 and also binds, although less strongly, to the HLA-B7 antigen. Affinity studies by Scatchard analysis (provided by the company) showed that the antibody has a higher affinity for HLA-B27. After intensive washing, cells were resuspended and incubated with secondary antibody (R-PE donkey anti-mouse; Jackson Immunoresearch, West Grove, USA) for $20 \mathrm{~min}$. Cells were fixed with $1 \%$ formaldehyde. Fluorescence was assayed using flow cytometry (LSRFortessa SORP, BD Biosciences, San Jose, USA); PE was excited by a $561 \mathrm{~nm}$ laser and emission was collected with a $585 / 15$ bandpass filter. Data were analysed using FlowJo v10.1 (FlowJo, Ashland, Oregon, USA). Cells were first gated on forward scatter and side scatter; subsequently, HLA-B27 positivity was determined by looking at $\mathrm{PE}$ signal versus side scatter (ATDC5) or forward scatter (hPDC).

\section{RNA extraction and quantitative RT-PCR}

For RNA isolation, samples were digested in Trizol (Thermo Fisher Scientific, Ghent, Belgium). RNA was isolated using the Nucleospin RNA II kit (Macherey-Nagel, Düren, Germany) according to the manufacturer's protocol. Complementary DNA was synthesised from $500 \mathrm{ng}$ of RNA using the RevertAid $\mathrm{H}$ minus First Strand cDNA synthesis kit (Fermentas,
St-Leon-Rot, Germany). Gene expression was quantified by SYBR green (Fermentas) RT-PCR using the Corbett Rotor-Gene 6000 (Corbett Research, Westburg, Leusden, The Netherlands). Primers were designed for HLA-B27 and markers of chondrogenesis (Aggrecan $(A C A N)$, type II collagen (COL2), COLX, SOX9 and matrix metalloproteinase $13(M M P 13)$ ) and osteogenesis (osteocalcin $(O C N)$, osterix $(O S X)$, bone sialoprotein $(B S P)$, alkaline phosphatase (ALP) and RUNX2). HPRT1 was used as a housekeeping gene. Primer sequences are available on request.

\section{Colorimetric tests}

After washing with PBS, cells were fixed in 95\% ice-cold methanol for $30 \mathrm{~min}$ at $4^{\circ} \mathrm{C}$. After washing intensively with distilled water, the cells were stained in either Alcian blue $(0.1 \%$ Alcian Blue 8GX (Sigma-Aldrich) in $0.1 \mathrm{M} \mathrm{HCl}$ pH 0.2), Safranin O (Klinipath, Olen, Belgium), Alizarin red (1\% Alizarin Red S (Sigma-Aldrich) in water $\mathrm{pH} 4.2$ ) or Sirius red $(0.1 \%$ Direct Red 80 (Sigma-Aldrich) in a saturated aqueous solution of picric acid). To remove unbound staining, cells were washed with distilled water until the washing solution remained colourless.

\section{ALP activity assay}

Cells were resuspended in Pierce IP Lysis Buffer (Thermo Fisher Scientific). Alkaline phosphatase (ALP) activity was measured using the BluePhos Microwell Phosphate Substrate system (KPL, Gaithersburg, Maryland, USA) following the manufacturer's instructions.

\section{Collagen antibody induced arthritis in mice}

Collagen antibody induced arthritis (CAIA) was induced in HLA-B27 transgenic and WT C57BL/6 male mice ( $\mathrm{n}=10$ in each group) using the ArthritoMab Antibody Cocktail kit for C57BL/6 (MD Biosciences, Zürich, Switzerland) following the manufacturer's instructions (with approval of the ethical committee UZ Leuven, ML 7770). Four microgram of antibody cocktail was injected intraperitoneally followed by a boost given after 3 days with an intraperitoneal injection of LPS. Clinical scoring was performed twice a week. The following scoring system was used: severe ankle inflammation: five points, moderate ankle inflammation: three points, mild ankle inflammation: one point, inflammation metatarsophalangeal joint: one point, inflammation interphalangeal joint: one point. The sum of the scores of the four paws was calculated for each time point. $\mu \mathrm{CT}$ scans (SkyScan 1076, Bruker, Evere, Belgium) from the hind paws were performed at baseline and 3 and 6 weeks after disease induction. The volume of the osteophytes at the navicular bone of the hind paws was measured by CTAn software (Bruker).

\section{Statistics}

GraphPad Prism was used to perform statistical analyses. Normality of data distribution was checked by graphs and normality tests. Parametric (t-test, ANOVA) or non-parametric tests (Mann-Whitney, Kruskal-Wallis) were used accordingly. For experiments with multiple variables 
and/or repeated measurements 2-way ANOVA was used. In case of unequal variance, data were log transformed or rank transformed. A 2-way ANOVA included assessment of time-intervention interaction, time and intervention.

\section{RESULTS}

\section{In vitro experiments}

An important part of the new bone formation in AS is hypothesised to be endochondral. ${ }^{12}$ Therefore, we used different in vitro systems mimicking this processATDC5 cell micromasses, hPDC micromasses and hPDCs in monolayer and limb bud cell micromassesto study the potential role of HLA-B27 in new bone formation in AS.

\section{HLA-B27 overexpression does not influence endochondral} bone formation in ATDC5 micromasses

The first model we used was ATDC5 cell micromasses in which this cell line undergoes a chondrogenic process followed by a mineralization phase when treated with chondrogenic and/or mineralization media. Micromasses were set up with ATDC5 cells transduced with lentiviral vectors expressing HLA-B27 or HLA-B7 (control vector). Expression of HLA-B27 and HLA-B7 was successful as shown by flow cytometry using an antibody that detects both human molecules but with higher affinity for HLA-27 (figure 1A). Samples for RNA extraction, followed by quantitative RT-PCR, and colorimetric tests were taken at days 1, 7, 14 and 21. As shown in figure 2A, there was no difference in expression of the cartilage markers between the HLA-B27 and the HLA-B7 overexpressing micromasses. The experiment was performed in triplicate and this was a consistent finding. There was also no difference observed in the colorimetric assessment of proteoglycan synthesis (Alcian blue (figure 2B) - Safranin O (data not shown)), mineralization (Alizarin red) (figure 2B) and collagens (Picrosirius red (data not shown)) of the HLA-B27 $7^{+}$and the HLA-B $7^{+}$micromasses.

\section{HLA-B27 overexpression does not influence chondrogenesis in hPDC micromasses}

The second model we used was micromasses set up with HLA-B27 and HLA-B7 transduced hPDCs. When treated with chondrogenic media, hPDC micromasses are also known to undergo chondrogenesis. ${ }^{14}$ The transduction was efficient as shown by FACS, although expression levels of HLA-B27 and B7 were lower than seen in the ATDC5 cells (figure 1B). Samples for RNA extraction, followed by quantitative RT-PCR, and Alcian blue staining were taken at days 1, 7 and 14. Similar to the observations in the ATDC5 micromasses, the expression of chondrogenesis markers (ACAN, COL2 and SOX9 as markers of early chondrogenesis and $C O L 10$ and RUNX2 as markers of late chondrogenesis) was not different between the HLA-B27 and HLA-B7 transduced hPDC micromasses (figure 3A). Only the expression of COL2 was significantly lower in the HLA-B27 $7^{+}$micromasses compared with the HLA-B7 $7^{+}$ micromasses (2-way ANOVA, Sidak post hoc $\mathrm{p}=0,0009$ ) at day 14 and the expression of RUNX2 was significantly higher in HLA-B27 $7^{+}$micromasses compared with HLA-B7 ${ }^{+}$micromasses (2-way ANOVA, Sidak post hoc $\mathrm{p}=0,0138$ ) at day 1 (figure $3 \mathrm{~A}$ ). There was no difference seen in the Alcian blue staining of the two types of micromasses (figure 3B). We therefore conclude that chondrogenesis in hPDC micromasses is not affected by presence of HLA-B27.

\section{HLA-B27 overexpression does not influence osteogenesis} in hPDCs

To study osteogenesis in hPDCs, cells were cultured in monolayers for 28 days. hPDCs are known to undergo osteogenesis when treated with osteogenic media. ${ }^{14}$ Samples for RNA extraction, followed by quantitative RT-PCR, ALP activity assay and Alizarin red staining, were taken at days 1, 7, 14, 21 and 28. There was no difference in expression of osteogenesis markers (OCN, ALP and RUNX2) between HLA-B27 $7^{+}$and HLA-B $7^{+}$hPDCs (figure 4A). OSX and BSP were not expressed in this set up. The ALP activity assay showed no significant difference between HLA-B27 $7^{+}$and HLA-B $7^{+}$hPDCs (figure 4B). Alizarin red staining did not differ between the two conditions (figure 4C). Collectively, we conclude that HLA-B27 does not directly influence osteogenesis in hPDCs.

HLA-B27 does not influence endochondral bone formation in limb bud cell micromasses

The last model we used was HLA-B27 transgenic and WT C57BL/6 limb bud micromasses. Limb bud cell micromasses are known to undergo endochondral bone formation in vitro when treated with chondrogenic media. ${ }^{16}$ Samples for RNA extraction, followed by quantitative RT-PCR, and colorimetric tests (Alizarin red and Alcian blue) were taken at days 1, 3,6 and 9. All embryos used in this experiment were from the same litter to make sure that the limb bud cells were exactly the same age as this might be important for the further course of differentiation. Chondrogenesis in this model is less straight forward than in the other models as shown in figure 5. Early chondrogenesis marker expression ( Col2 and $5 \circ \times 9)$ was decreased from day 1 onwards which suggests a rapid acceleration of in vivo initiated chondrogenic differentiation from day 1 already, except for Acan which expression peaked at day 6 (except one WT embryo that peaks at day 9). Col10 expression-as a marker of late chondrogenesis-peaked already on day 3 for all samples, whereas the other late chondrogenesis markers (Runx2 and Mmp13) peaked at day 9 . This course of gene expression was a consistent finding in different limb bud micromass experiments (data not shown). Altogether, there was no striking difference between the course of chondrogenesis gene expression in HLA-B27 $7^{+}$and WT micromasses at the molecular level (figure 5). The Alizarin red and Alcian blue staining were also similar (data not shown). 
A. ATDC5 Wild-type ATDC5 HLA-B 7 transduced ATDC5 HLA-B27 transduced

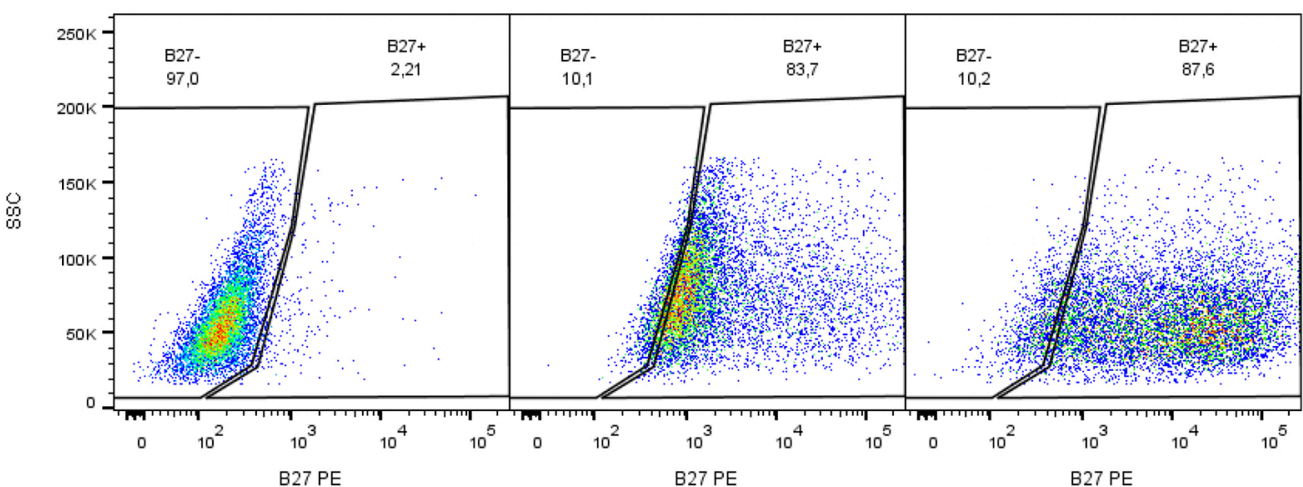

B.

hPDC Wild-type

hPDC HLA-B7 transduced

hPDC HLA-B27 transduced

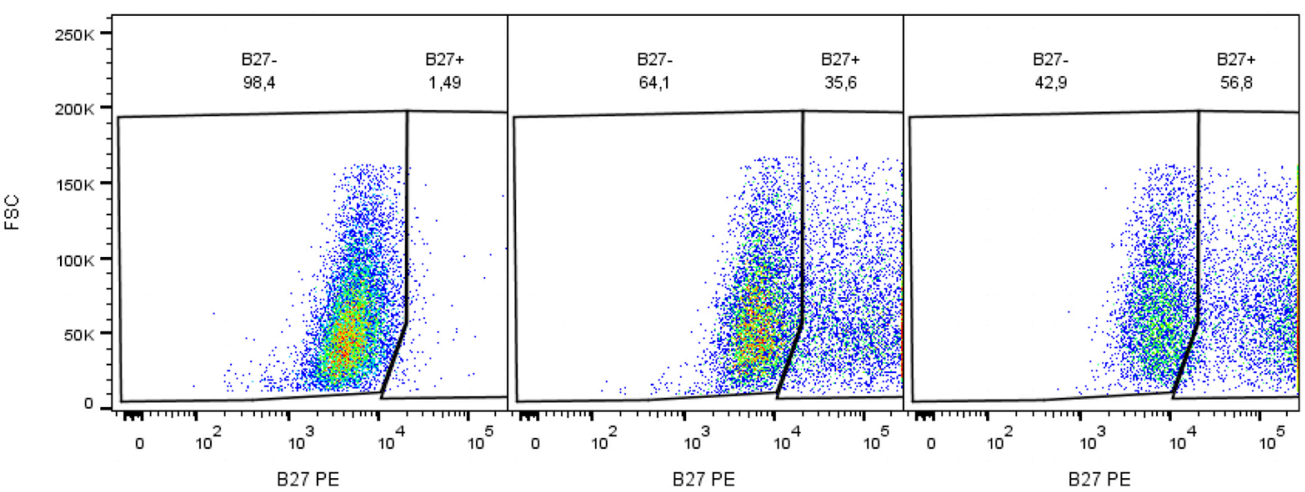

C.
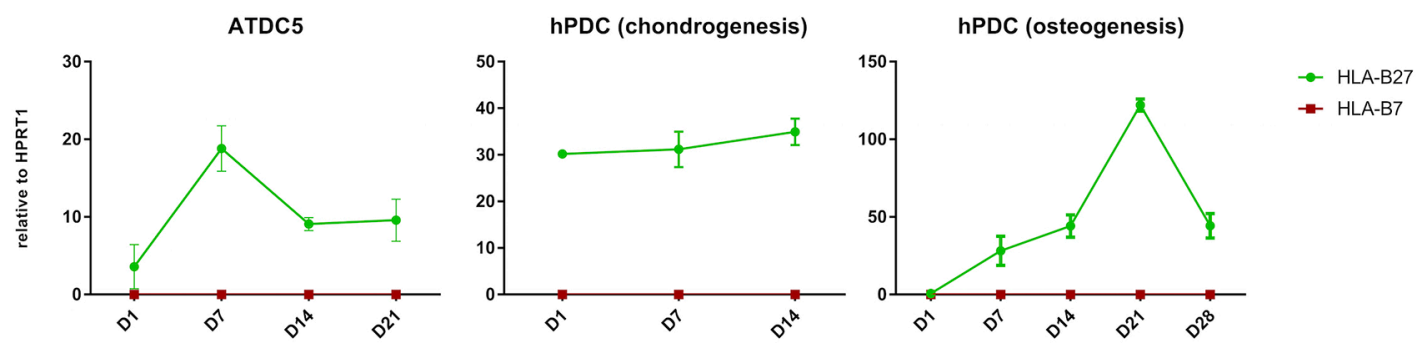

Figure 1 Flow cytometry analysis of HLA-B27 and HLA-B7 transduced cells. (A) ATDC5 cells. (B) hPDCs were stained with an HLA-B27 antibody and subsequently with a PE-linked secondary antibody. There is a very strong cross-reactivity known between HLA-B7-positive cells and the HLA-B27 antibody, as the antibody used binds to both HLA-B27 and HLA-B7. Cells were first gated on forward scatter and side scatter; subsequently, HLA-B27/B7 positivity was determined by looking at PE signal versus side scatter (ATDC5) or forward scatter (hPDC). The percentage of HLA-B27-positive and HLA-B27-negative cells is noted above the plots. (C) Expression of HLA-B27 measured by quantitative RT-PCR, with an HLA-B27-specific primer, at the different time point during differentiation of ATDC5 cells, hPDCs during chondrogenesis and hPDCs during osteogenesis. FSC, forward-scattered; SSC, side-scattered.

\section{In vivo experiment}

For the in vivo part, we induced CAIA in HLA-B27 transgenic and WT C57BL/6 mice ( $\mathrm{n}=10$ in each group). The CAIA model is characterised by the development of synovitis, enthesitis and late-stage new bone formation which makes it a valuable model to study AS.

\section{HLA-B27 increases severity of arthritis in the CAIA model} To investigate the influence of HLA-B27 on inflammation in the in vivo CAIA model, clinical scores were performed twice a week during 6 weeks. The HLA-B27 transgenic mice $(n=10)$ showed more severe arthritis of the paws compared with the WT mice ( $\mathrm{n}=10$ at start, one mice died at day 10 and was excluded from analysis). The initiation and duration of the inflammation did not differ between the two groups, but the intensity of inflammation was significantly higher at days 7, 10 and 14 in the group of the HLA-B27 transgenic mice compared with the WT mice (2-way ANOVA, Sidak post hoc $\mathrm{p}=0.0469$, $\mathrm{p}<0.0001$ and $\mathrm{p}=0.0056$, respectively) (figure $6 \mathrm{~A}$ ). The weight of the mice was the same in the two groups (figure 6B) which suggests more local inflammation without pronounced impact on the general health of the mice. 
A.

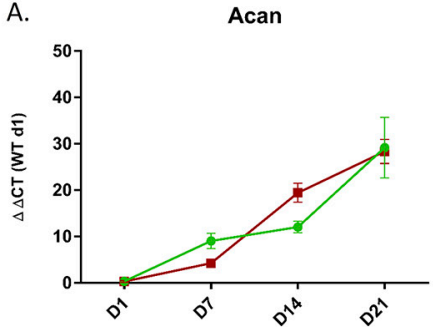

Col2

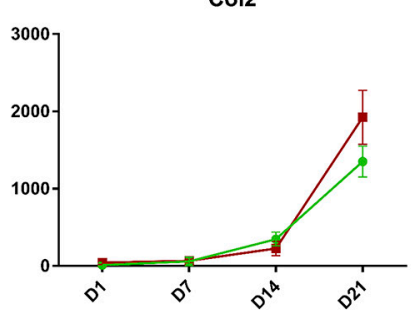

Col10

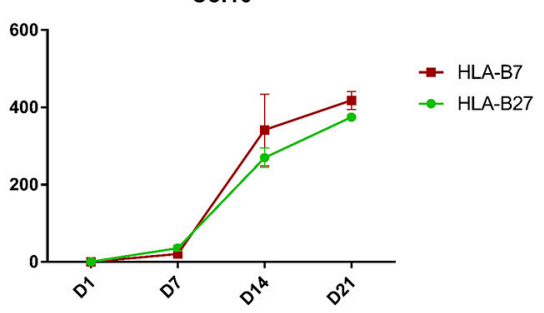

B.
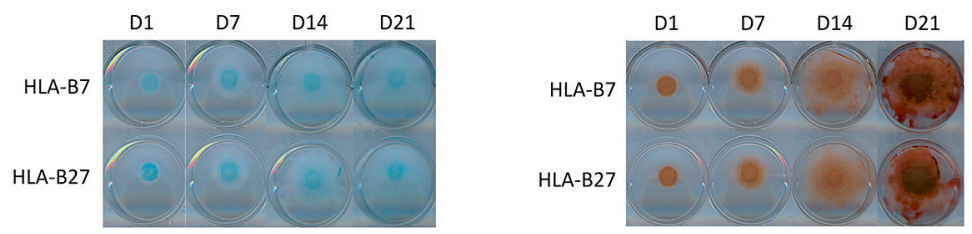

Figure 2 Endochondral bone formation in HLA-B27 transduced ATDC5 micromasses. (A) Expression of Acan, Col2 and Col10 measured by quantitative RT-PCR in the HLA-B27 and HLA-B7 ATDC5 micromasses. Statistical analysis was performed by two-way ANOVA. $p<0.05$ was considered as statistically significant. Data points are shown as average and $S E M(n=3)$. (B) Alcian blue and Alizarin red staining at the different time points. Acan, Aggrecan; Col2, Collagen type II; Col10, Collagen type X.

\section{HLA-B27 does not influence new bone formation in the} CAIA model

In the CAIA model, osteophytes develop in the post-inflammatory phase. To study the role of HLA-B27 in new bone formation in the in vivo CAIA model, the volume of the osteophyte(s) at the navicular bone of the hind paws was measured by $\mu \mathrm{CT}$. In figure $6 \mathrm{C}$, volumes of left and right hind paws were summed. All paws except two in the HLA-B27 transgenic group and four in the WT group developed osteophytes. $\mu \mathrm{CT}$ analysis by CTAn showed no increased new bone formation when HLA-B27 transgenic mice were compared with WT (figure 6C). There was no significant correlation between the peak inflammation score and the volume of osteophytes (Pearson correlation, $\mathrm{R}^{2}=0.1149, \mathrm{p}=0.1557$ ) (figure $6 \mathrm{D}$ ).

\section{DISCUSSION AND CONCLUSION}

The link between AS and HLA-B27 has been known already for more than 40 years. ${ }^{45}$ Despite this, little is known about the possible role of HLA-B27 in new bone formation and ankylosis, one of the hallmarks of AS. Most research in AS, and particularly the research focusing on the role of HLA-B27 in the pathogenesis, focuses on
A.

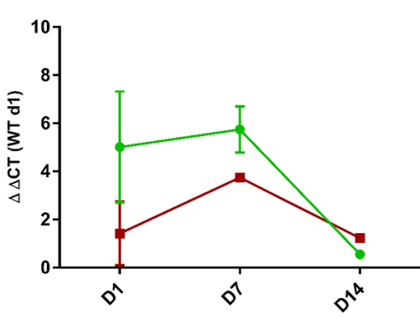

RUNX2

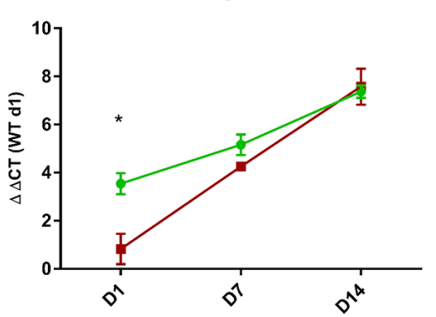

COL2

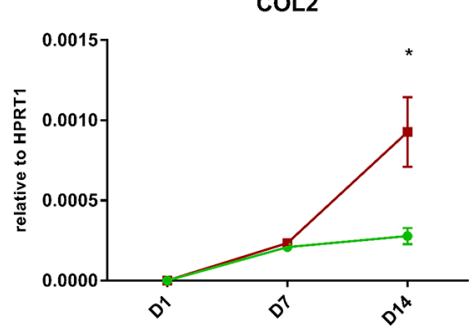

sox9

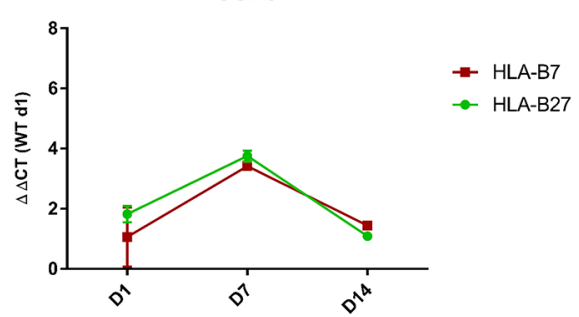

COL10

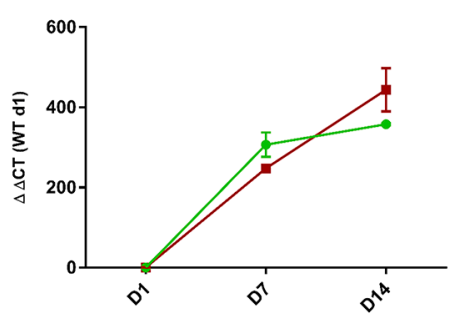

B.

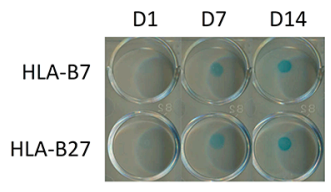

Figure 3 Chondrogenesis in HLA-B27 transduced hPDC micromasses. (A) Expression of ACAN, COL2, COL10, RUNX2 and SOX9 measured by quantitative RT-PCR in the HLA-B27 and HLA-B7 hPDC micromasses. Statistical analysis was performed by two-way ANOVA. $p<0.05$ was considered as statistically significant. Data points are shown as average and SEM ( $n=3)$. (B) Alcian blue staining at the different time points. WT, wild-type. 

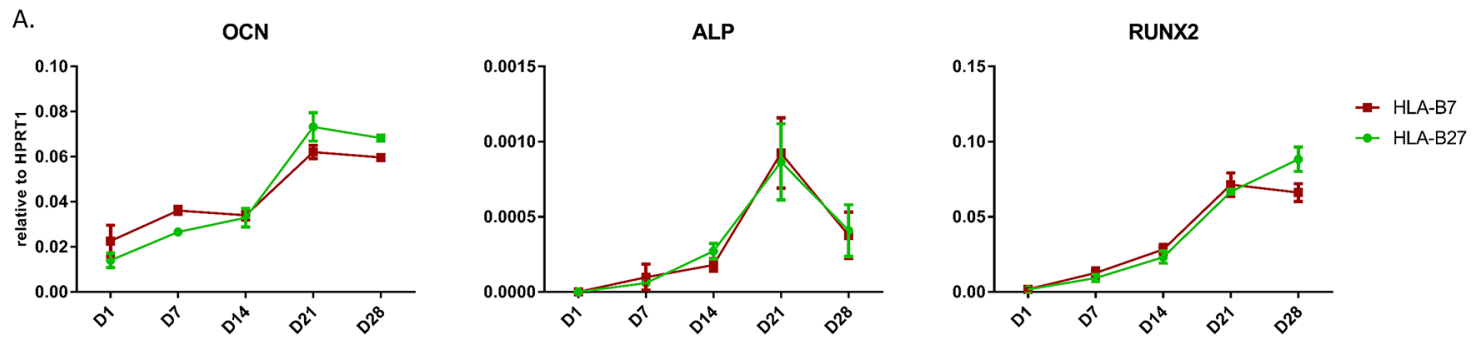

B.

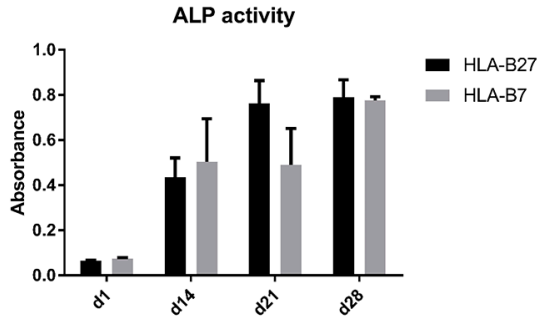

C.

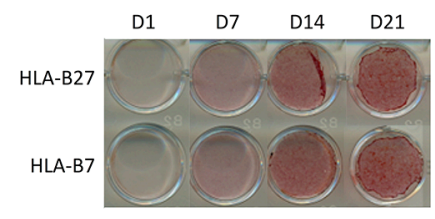

Figure 4 Osteogenesis in monolayer HLA-B27 transduced hPDCs. (A) Expression of OCN, ALP and RUNX2 measured by quantitative RT-PCR in the HLA-B27 and HLA-B7 hPDCs. Statistical analysis was performed by two-way ANOVA. $p<0.05$ was considered as statistically significant. (B) ALP activity assay. Data points are shown as average and SEM $(n=3)$. (C) Alizarin red staining at the different time points. ALP, alkaline phosphatase; OCN, osteocalcin.

inflammation-the second hallmark of AS and leading cause of acute signs and symptoms of the disease. We set up this study to investigate the possible role of HLA-B27 in new cartilage and bone formation. The data obtained in our study do not show evidence for a direct role for HLA-B27 in new cartilage or bone formation. The presence of HLA-B27 does not seem to affect endochondral bone formation in vitro or in vivo, neither in a positive nor in a negative way.
Ideally, human samples are used to study disease pathogenesis as their translational value is much higher than samples from in vitro models. As it is very difficult to obtain samples from patients with AS, in particular, those with early disease-due to ethical concerns as well as due to the long time window to develop ankylosis-the use of in vitro models was necessary to investigate the role of HLA-B27 in new bone formation in this study. As no single in vitro model perfectly mimics the process of
A.

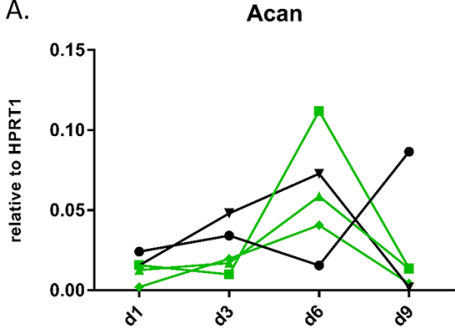

Sox9

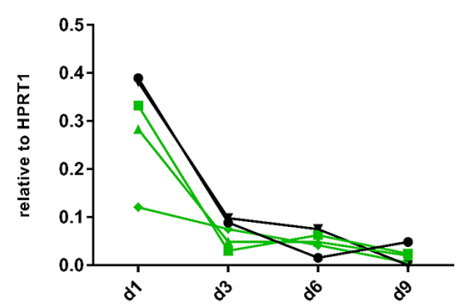

Col2

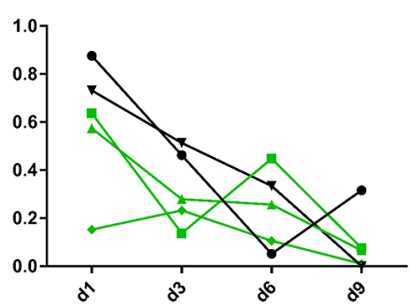

Runx2

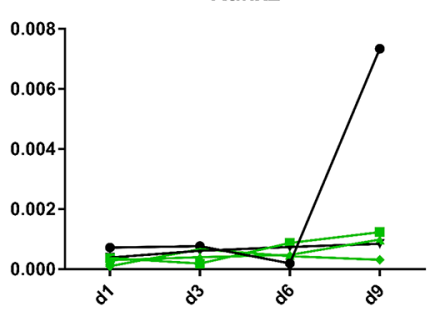

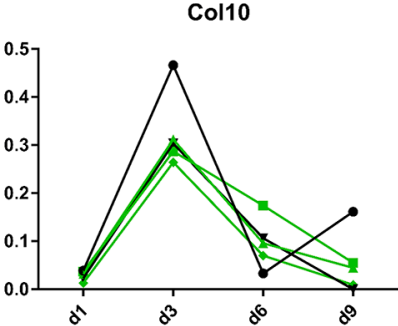

$\rightarrow 17$

$\rightarrow 16$

$-15$

- 14

- 13

HLA-B27 ${ }^{+}$

WT

Figure 5 Endochondral bone formation in HLA-B27 and WT limb bud micromasses. Expression of Acan, Col2, Col10, Sox9, Runx2 and Mmp13 measured by quantitative RT-PCR in the HLA-B27 and WT limb bud micromasses. Each number represents an embryo - green samples are HLA-B27-positive; black samples are WT. WT, wild-type. 
A.

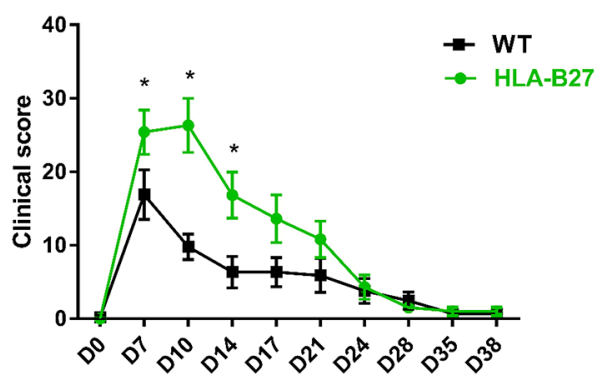

C.

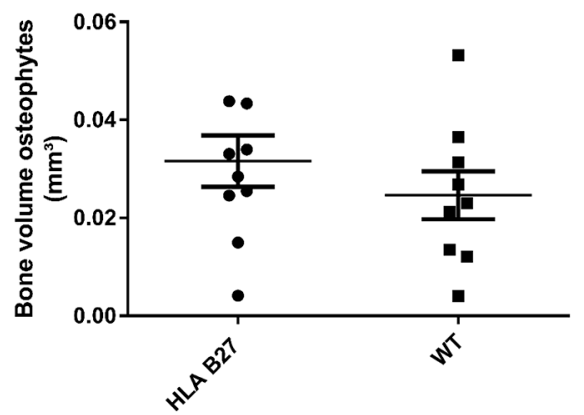

B.

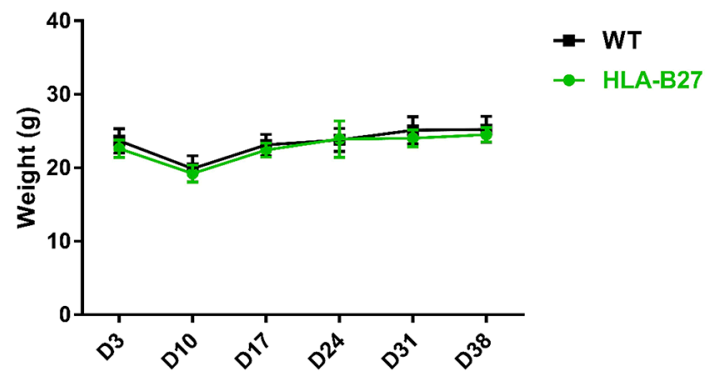

D.

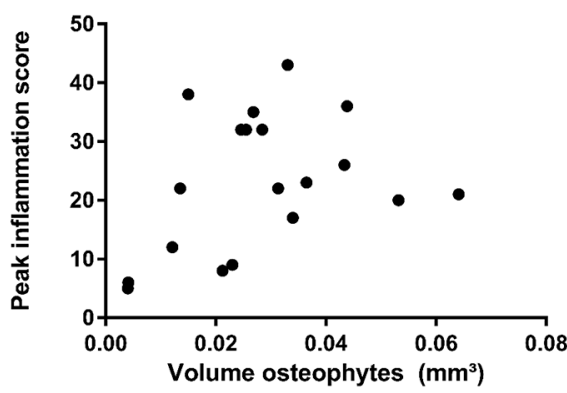

Figure 6 CAIA model in HLA-B27 transgenic versus WT C57BL/6 mice. (A) Clinical score of mice at different time points. Data points are shown as average and SEM. Statistical analysis was performed by two-way ANOVA, Sidak post hoc. $p<0.05$ was considered as statistically significant. (B) Weight of mice at different time points. Data points are shown as average and SEM. (C) Measurement of osteophyte volume at the navicular bone of the left and right hind paw at 6 weeks after disease induction. Data points are the sum of the volume of the osteophytes at the left and at the right paw from the same mouse. Data points are shown as average and SEM. Statistical analysis was performed by unpaired t-test. $p<0.05$ was considered as statistically significant. (D) Correlation between peak inflammation score and osteophyte volume (sum of left and right paw).

human disease, we chose to combine multiple modelsall of them with their own strengths and weaknesses. Research from animal models provides strong evidence that a large part of the new bone formation in ankylosis is due to endochondral bone formation. ${ }^{17}$ Therefore, we chose to use in vitro models mimicking this process. Endochondral bone formation is characterised by an initial phase of chondrogenesis followed by a terminal mineralization phase with attraction of osteoblast precursor cells leading to bone replacement. ${ }^{18}$ The first model used is the ATDC5 micromass model. The presence of HLA-B27 in ATDC5 micromasses does not seem to influence the process of endochondral bone differentiation. The ATDC5 micromass system is a well-known in vitro model mimicking endochondral bone formation. It is a very robust model with an extensive expertise in our laboratory. However, the ATDC5 cell line is a mouse cell line and might lack expression of important human molecules on the one hand and/or express mouse molecules on the other hand that could influence the behaviour of HLA-B27. One such a molecule is $\beta 2$ microglobulin $(\beta 2 \mathrm{~m})$, a molecule that binds to the HLA-B27 heavy chain in the endoplasmic reticulum. Human $\beta 2 \mathrm{~m}$ stabilises the HLA-B27 heavy chain when expressed at the cell surface. Overexpression of additional human $\beta 2 \mathrm{~m}$ in HLA-B27/human $\beta 2 \mathrm{~m}$ transgenic rats promotes arthritis and spondylitis, ${ }^{19}$ suggesting an important role for this human molecule in the pathogenesis of AS. Mouse $\beta 2 \mathrm{~m}$, however, could inhibit the possible inflammatory and osteogenic properties of HLA-B27 as it is seen that HLA-B27 transgenic mice do not show inflammatory disease $^{1520}$ whereas HLA-B27 expression in a mouse $\beta 2 \mathrm{~m}$-deficient mouse model induces inflammatory arthritis of the hind paws and nail changes. ${ }^{21}$

To overcome this issue, we studied another micromass model using human cells, more specifically hPDCs. These cells do express human $\beta 2 \mathrm{~m}$. This model only mimics the chondrogenic part of endochondral bone formation. In the monolayer experiments, we also tested direct osteogenic differentiation. Also in hPDC models, the presence of HLA-B27 does not influence new cartilage or bone formation. hPDC micromasses show less pronounced chondrogenesis than ATDC5 micromasses which is reflected in the course and magnitude of chondrogenic gene expression. The combination of gene expression and colorimetric tests, however, make it a valuable model. As endochondral bone formation is an essential process in developing long bones, the limb bud micromasses are a logic model to study this process in vitro. Remarks for the use of this model in our study are the fact that these cells are also derived from mice and thus express mouse $\beta 2 \mathrm{~m}$ and the fact that gene expression is highly variable between different samples. The latter is also the reason why gene expression is presented per embryo and is not 
pooled as average. Despite the limitations of the individual models, the strength of this study is the consistent finding that in all the used models - either human or mouse- the presence of HLA-B27 does not seem to influence new cartilage and bone formation.

The in vitro models could also be too simplistic and miss crucial molecules to mimic the real in vivo setting of new cartilage and bone formation. However, this rather simple set up was a conscious choice to study the direct role of HLA-B27 in chondrogenic/osteogenic cell differentiation without influence of possibly interfering molecules. We therefore also purposefully chose not to trigger cells with inflammatory cytokines. Furthermore, consistent with the in vitro findings, the presence of HLA-B27 did also not influence new bone formation in the in vivo model we used. In this study, we chose the CAIA model to study new bone formation in vivo. New bone formation, present as osteophytes, occurs in the post-inflammatory phase in this model and is present in almost every mouse. Intriguingly, there was more inflammation seen in the HLA-B27 transgenic animals compared with the controls. Bone formation, however, did not differ significantly between the groups. This finding might be suggestive that HLA-B27 does not play a role in new bone formation in AS but that its specific and unique properties, including misfolding and cell surface dimer expression, may be an additional driving factor in different types of inflammation.

There are several plausible explanations for the negative results of our study. First, it is possible that HLA-B27 is only necessary to trigger the disease and does not play a role in the further development of AS, in particular, in those processes that lead to radiographic changes.

Second, it is plausible that HLA-B27 only plays a role in the inflammatory part of the AS pathogenesis. In the last decade, much research has been focused on the link between HLA-B27 and AS. Several interesting hypotheses are postulated-like the unfolded protein response and the free heavy chain hypotheses -all mainly focusing on inflammation and not on new bone formation. None of these hypotheses perfectly fills the knowledge gap about the exact role of HLA-B27 in AS. Probably, a very complex interaction of all these factors may lead to the development of AS in HLA-B27-positive individuals. Because inflammation and new bone formation in AS are supposed to be at least partially coupled processes, it is possible that HLA-B27 plays an indirect role-by triggering inflammation-in the development of new bone in AS rather than a direct role. The current paradigm suggests that fatty lesions are gradually replacing areas of active inflammation in the spine and sacroiliac joints. This change in microenvironment is permissive for the further evolution towards new bone formation and ankylosis, although the suggested sequence of inflammation, fat metaplasia and bone formation is still debated. As new syndesmophytes can also be seen in sites without documentation of prior inflammation, it seems that inflammation and new bone formation are linked but to a large extent also uncoupled processes. ${ }^{22}{ }^{23}$ Also in favour of the hypothesis that HLA-B27 is indirectly linked to new bone formation is the fact that spinal hyperostosis per se, such as seen in patients with diffuse idiopathic skeletal hyperostosis, is not linked to HLA-B27.

Third, it is possible that the presence of the HLA-B27 molecule alone is not sufficient to influence new bone formation. Maybe a more complex milieu is needed to induce new cartilage and bone. A possible contributing factor could be human $\beta 2 \mathrm{~m}$, as discussed above. Also cytokines could be the missing link between HLA-B27 and new bone formation. Another additional factor that could be the missing link between HLA-B27 and new bone formation is mechanical loading. Mechanical loading plays an important role in new bone formation in AS as shown by Jacques $e t a l^{24}$ who induced CAIA in DBA/ 1 mice. Subsequently half of the mice were tail suspended for 28 days, to prevent mechanical loading on hind paws, and half were kept in normal cages. In tail suspended mice, osteophytes were significantly smaller compared with control mice. ${ }^{24}$ There is also a small study showing a correlation between the extent of bone pathology and presence of HLA-B27 in patients with enthesitis at the plantar fascia. ${ }^{25}$ As biomechanical loading does not cause new bone formation in every individual, it is possible that presence of HLA-B27 links biomechanical stress to development of new bone formation in AS and/or vice versa. To further investigate this research question, dynamic in vitro models with HLA-B27-positive cells are necessary by contrast with the static models used in this study. Recent evidence shows that presence of HLA-B27 in humans affects the serum levels of key regulators of bone homeostasis, such as DDK1, sclerostin and IHH. ${ }^{26}$ The fact that these altered serum levels were seen in HLA-B27-positive AS patients as well as in HLA-B27-positive healthy individuals also suggests the need of an extra trigger to induce the disease. It can currently not be excluded that HLA-B27-positive individuals are more prone to an enhanced reaction of bone to stress, like inflammation or mechanical loading for example.

Finally, it is possible that higher levels of HLA-B27 expression are needed to influence bone formation. In the HLA-B27/human $\beta 2 \mathrm{~m}$ transgenic rats, it is known that the degree of susceptibility for spondyloarthritis-like lesions correlates with the level of HLA-B27 transgene expression at the mRNA and protein levels, as kind of a 'threshold' effect, where a certain level of HLAB27/ human $\mathrm{b} 2 \mathrm{~m}$ expression must be achieved to obtain a disease phenotype. ${ }^{27}$ The expression of HLA-B27 in patients with AS also appears to be higher compared with the expression in HLA-B27-positive healthy controls or family members, ${ }^{28}{ }^{29}$ with no difference in expression of total MHC class I molecules between these groups. ${ }^{28}$ Only in the ATDC5 micromasses was the HLA-B27 expression was high, but the molecule's biology could be functionally disturbed by mouse $\beta 2 \mathrm{~m}$. In the other models-hPDCs, limb bud cells and CAIA model-HLA-B27 expression 
was present in about $50 \%$ of the cells, which mimics the human heterozygote situation.

In conclusion, the results of our study do not argue for a direct role of HLA-B27 on new cartilage and bone formation in AS. If HLA-B27 plays a role in ankylosis, it will probably be an indirect role with HLA-B27 contributing to inflammation. Nevertheless, all negative experiments, even if confirmed by different approaches, should be interpreted with caution and only confirm the absence of evidence rather than a clear proof. Future research, focusing on the specific working mechanism of HLA-B27 on the one hand and the exact link between inflammation and new bone formation on the other, is necessary to investigate if HLA-B27 plays an indirect role in new bone formation.

Acknowledgements Barbara Neerinckx is the recipient of an Aspirant PhD fellowship from the Flanders Research Foundation (FWO Vlaanderen). This project was funded by the FW0 grant G.0946.14.

Contributors BN and RL initiated the study and wrote the manuscript. BN performed experiments and developed reagents. SK and JS developed and optimized the used plasmids. All authors were involved in drafting the article or revising it critically for important intellectual content. All authors approved the final version to be published.

Competing interests None declared.

Provenance and peer review Not commissioned; externally peer reviewed.

Data sharing statement All data supporting the findings of this study are available within the article and its supplementary files, or available from the authors upon request.

Open Access This is an Open Access article distributed in accordance with the Creative Commons Attribution Non Commercial (CC BY-NC 4.0) license, which permits others to distribute, remix, adapt, build upon this work non-commercially, and license their derivative works on different terms, provided the original work is properly cited and the use is non-commercial. See: http://creativecommons.org/ licenses/by-nc/4.0/

(C) Article author(s) (or their employer(s) unless otherwise stated in the text of the article) 2017. All rights reserved. No commercial use is permitted unless otherwise expressly granted.

\section{REFERENCES}

1. Machado P, Landewé R, Braun J, et al. Both structural damage and inflammation of the spine contribute to impairment of spinal mobility in patients with ankylosing spondylitis. Ann Rheum Dis 2010;69:1465-70.

2. Lories RJ, Luyten FP, de Vlam K. Progress in spondylarthritis. mechanisms of new bone formation in spondyloarthritis. Arthritis Res Ther 2009;11:221.

3. Breban M. Genetics of spondyloarthritis. Best Pract Res Clin Rheumatol 2006;20:593-9.

4. Schlosstein L, Terasaki PI, Bluestone R, et al. High association of an HL-A antigen, W27, with ankylosing spondylitis. N Engl J Med 1973;288:704-6.

5. Brewerton DA, Hart FD, Nicholls A, et al. Ankylosing spondylitis and HL-A 27. Lancet 1973;1:904-7.

6. Colbert RA, DeLay ML, Klenk El, et al. From HLA-B27 to spondyloarthritis: a journey through the ER. Immunol Rev 2010;233:181-202.

7. Bowness P. HLA-B27. Annu Rev Immunol 2015;33:29-48.
8. Ramiro S, Stolwijk C, van Tubergen A, et al. Evolution of radiographic damage in ankylosing spondylitis: a 12 year prospective follow-up of the OASIS study. Ann Rheum Dis 2015;74:52-9.

9. Atagunduz P, Aydin SZ, Bahadir C, et al. Determinants of early radiographic progression in ankylosing spondylitis. J Rheumatol 2010;37:2356-61.

10. Poddubnyy $\mathrm{D}$, Haibel $\mathrm{H}$, Listing J, et al. Baseline radiographic damage, elevated acute-phase reactant levels, and cigarette smoking status predict spinal radiographic progression in early axial spondylarthritis. Arthritis Rheum 2012;64:1388-98.

11. van Tubergen A, Ramiro S, van der Heijde D, et al. Development of new syndesmophytes and bridges in ankylosing spondylitis and their predictors: a longitudinal study. Ann Rheum Dis 2012;71:518-23.

12. Lories RJ, Haroon N. Bone formation in axial spondyloarthritis. Best Pract Res Clin Rheumatol 2014;28:765-77.

13. Lodewyckx L, Cailotto F, Thysen S, et al. Tight regulation of wingless-type signaling in the articular cartilage - subchondral bone biomechanical unit: transcriptomics in Frzb-knockout mice. Arthritis Res Ther 2012;14:R16.

14. De Bari C, Dell'Accio F, Tylzanowski P, et al. Multipotent mesenchymal stem cells from adult human synovial membrane. Arthritis Rheum 2001;44:1928-42.

15. Taurog JD, Lowen L, Forman J, et al. HLA-B27 in inbred and noninbred transgenic mice. Cell surface expression and recognition as an alloantigen in the absence of human beta 2-microglobulin. $J$ Immunol 1988;141:4020-3.

16. Underhill TM, Dranse HJ, Hoffman LM. Analysis of chondrogenesis using micromass cultures of limb mesenchyme. Methods Mol Biol 2014:1130:251-65.

17. Lories RJ, Matthys P, de Vlam K, et al. Ankylosing enthesitis, dactylitis, and onychoperiostitis in male DBA/1 mice: a model of psoriatic arthritis. Ann Rheum Dis 2004;63:595-8.

18. Lories RJ, Schett G. Pathophysiology of new bone formation and ankylosis in spondyloarthritis. Rheum Dis Clin North Am 2012;38:555-67.

19. Tran TM, Dorris ML, Satumtira N, et al. Additional human beta2microglobulin curbs HLA-B27 misfolding and promotes arthritis and spondylitis without colitis in male HLA-B27-transgenic rats. Arthritis Rheum 2006;54:1317-27.

20. Nickerson CL, Hanson J, David CS. Expression of HLA-B27 in transgenic mice is dependent on the mouse $\mathrm{H}-2 \mathrm{D}$ genes. J Exp Med 1990;172:1255-61.

21. Khare SD, Luthra HS, David CS. Spontaneous inflammatory arthritis in HLA-B27 transgenic mice lacking beta 2-microglobulin: a model of human spondyloarthropathies. J Exp Med 1995;182:1153-8.

22. Neerinckx B, Lories R. Mechanisms, impact and prevention of pathological bone regeneration in spondyloarthritis. Curr Opin Rheumatol 2017:1.

23. Neerinckx B, Lories RJ. Structural disease progression in axial spondyloarthritis: still a cause for concern? Curr Rheumatol Rep 2017;19:14

24. Jacques P, Lambrecht $\mathrm{S}$, Verheugen $\mathrm{E}$, et al. Proof of concept: enthesitis and new bone formation in spondyloarthritis are driven by mechanical strain and stromal cells. Ann Rheum Dis 2014;73:437-45.

25. McGonagle D, Marzo-Ortega $\mathrm{H}, \mathrm{O}^{\prime}$ Connor $\mathrm{P}$, et al. The role of biomechanical factors and HLA-B27 in magnetic resonance imaging-determined bone changes in plantar fascia enthesopathy. Arthritis Rheum 2002;46:489-93.

26. Aschermann S, Englbrecht M, Bergua A, et al. Presence of HLA-B27 is associated with changes of serum levels of mediators of the Wnt and hedgehog pathway. Joint Bone Spine 2016;83.

27. Taurog JD, Maika SD, Simmons WA, et al. Susceptibility to inflammatory disease in HLA-B27 transgenic rat lines correlates with the level of B27 expression. J Immunol 1993;150:4168-78.

28. Cauli A, Dessole G, Fiorillo MT, et al. Increased level of HLA-B27 expression in ankylosing spondylitis patients compared with healthy HLA-B27-positive subjects: a possible further susceptibility factor for the development of disease. Rheumatology 2002;41:1375-9.

29. Liu SQ, Yu HC, Gong YZ, et al. Quantitative measurement of HLA-B27 mRNA in patients with ankylosing spondylitis - correlation with clinical activity. J Rheumatol 2006;33:1128-32. 